\title{
New elements in old tools: Nucleosynthesis in $N$-body codes
}

\section{Guido R. I. Moyano Loyola*}

Centre for Astrophysics and Supercomputing

Swinburne University of Technology

PO Box 218, Melbourne, Australia

E-mail: gmoyanodastro.swin.edu.au

\section{Jarrod R. Hurley}

Centre for Astrophysics and Supercomputing

Swinburne University of Technology

PO Box 218, Melbourne, Australia

E-mail: jhurley@astro.swin.edu.au

\begin{abstract}
The main scientific goal of this project is to unravel for the first time the stellar assembly history of our Galaxy using an accurate dynamical and chemical approach. Of particular importance is to identify the early dissolution events of primordial stellar clusters and the overall impact on the chemical evolution of the Galaxy. We will do this by adding detailed nucleosynthesis models into the current state-of-the-art $N$-body code NBODY6. In this way we will obtain accurate predictions for the chemical evolution of the Galaxy and the evolution of stellar clusters, particularly the change of surface abundances from non-standard evolution, aspects which will be a novel implementation in the field.
\end{abstract}

XII International Symposium on Nuclei in the Cosmos,

August 5-12, 2012

Cairns, Australia

*Speaker. 


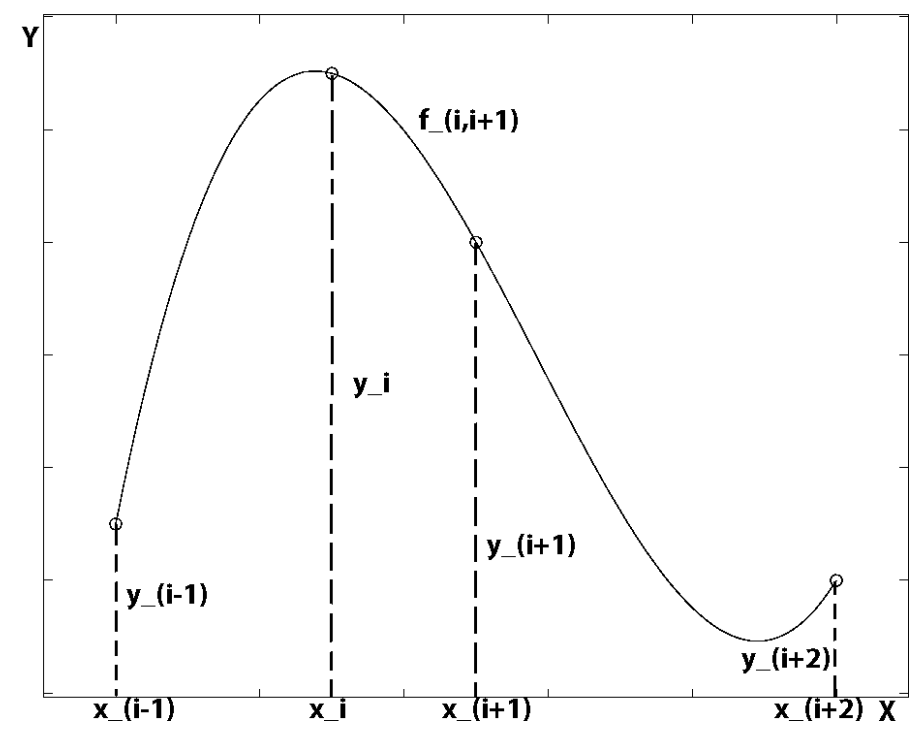

Figure 1: Schematic cartoon showing the four interpolating points used in the "cubic splines" scheme and the continuity of both, the first and second derivatives of the interpolating polynomial at the chosen points.

\section{Introduction}

Stellar evolution has been implemented in the direct $N$-body code NBODY6 (Aarseth 2003) since early 2000 as an analytical approach for Single Stellar Evolution (SSE: Hurley et al. 2000) and Binary Stellar Evolution (BSE: Hurley et al. 2002). This implementation has been incredibly successful in the past to replicate in an accurate way the evolution of a real star cluster.

Now that we are at the gates of major Galactic Archaeology projects (Freeman \& BlandHawthorn 2002) we need an improved approach that has not been attempted before: to track not only the kinematics and stellar evolution information but the chemical information as well in order to unravel the assembly history of our Galaxy.

\section{Method}

Our new approach will involve using tabulated models instead of the analytic functions implemented so far in NBODY6. For the required interpolation within these models we have chosen "cubic splines",

$$
f_{i-1, i}^{\prime \prime}\left(x_{i}\right)=f_{i, i+1}^{\prime \prime}\left(x_{i}\right)=k_{i},
$$

with the natural spline condition

$$
k_{1}=k_{n}=0 .
$$




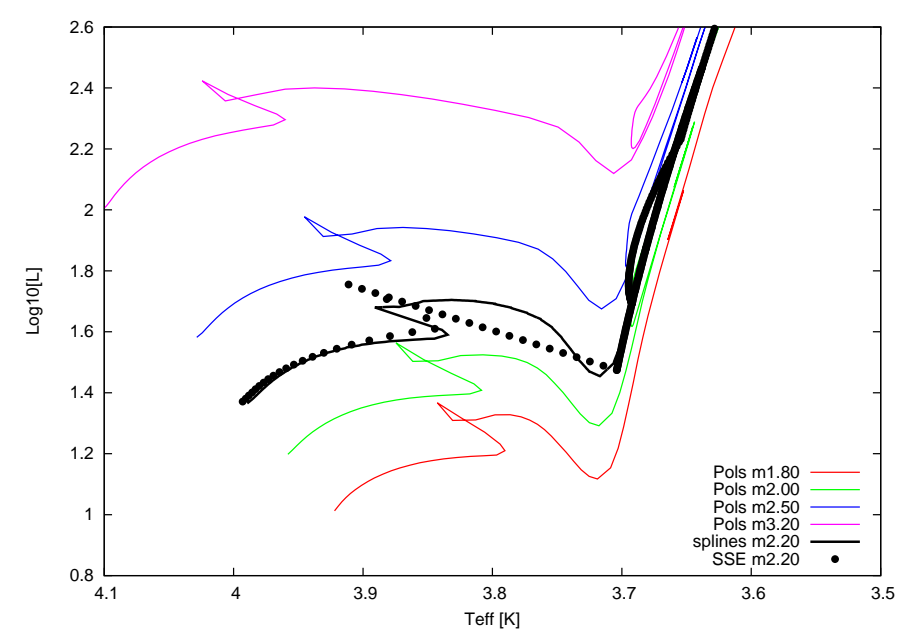

Figure 2: The colour solid lines represent the detailed models of Pols et al. (1998) for 1.8, 2.0, 2.5 and 3.2 $\mathrm{M}_{\odot}$, the black solid line is the interpolated curve obtained with cubic splines for $2.2 \mathrm{M}_{\odot}$ and the dotted line is the $2.2 \mathrm{M}_{\odot}$ benchmark done with SSE.

By interpolating within 4 models, as can be seen in Fig. 1, we obtain the values of structure and chemical composition for any particular mass for which we do not have the given table. We will also expand the approach to interpolate in metallicity using bi-cubic splines. NBODY6 will make frequents calls to the interpolating subroutine to retrieve the stellar parameters as required.

\section{Early results}

I have used the models of Pols et al. (1998) as the starting point to set up the cubic splines interpolating scheme. In order to compare the quality of the mentioned method I am using as a benchmark SSE. Fig. 2 shows the first results of the interpolation scheme for the main sequence.

The final goal is to use the models from the SINs group at Monash University and finally their complete grid which is the aim of the Mon $\chi$ ey project ${ }^{1}$.

\section{Galactic Archaeology}

The current capability of NBODY6 allows us to evolve and track stars that escape from a given stellar cluster. Once we have implemented the nucleosynthesis information within our code we will be able to trace back the building blocks of our Galaxy not only in phase space (i.e. coordinates and velocities) but in chemical space as well, and compare our results with the information that will be provided by missions such as GAIA ${ }^{2}$ and HERMES/GALAH ${ }^{3}$.

In this direction we are starting a new project called TraCD (Tracking Cluster Debris) in collaboration with Brad Gibson ${ }^{4}$ and Chris Flynn ${ }^{5}$ to track systems (i.e. single stars and binary systems)

\footnotetext{
${ }^{1}$ http://users.monash.edu.au/ johnl/monkey/

${ }^{2}$ http://sci.esa.int/science-e/www/area/index.cfm?fareaid=26

${ }^{3}$ http://www.aao.gov.au/HERMES/

${ }^{4}$ http://www.star.uclan.ac.uk/ bkg/

${ }^{5}$ http://www.astro.utu.fi/ cflynn/
} 


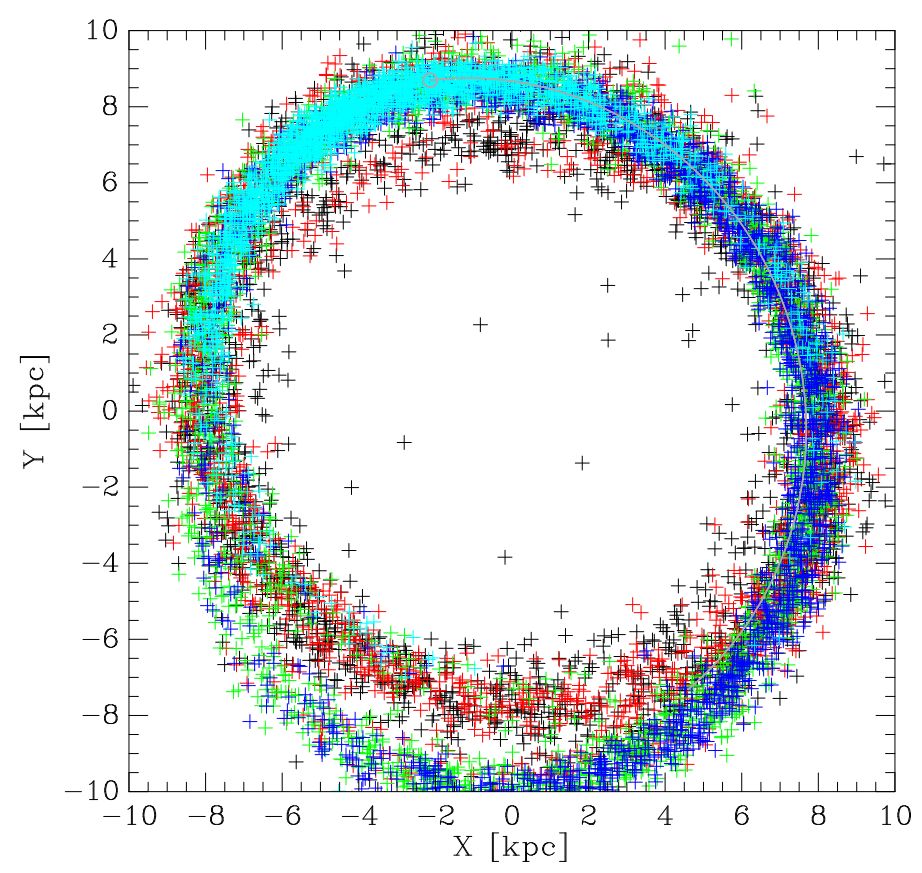

Figure 3: Evolution of escaper stars from an open cluster in the axi-symmetric potential of the Milky Way. The colour coding represents different times of escape (i.e. black are the first to leave, followed by red, green, dark blue and light blue), each group encompassing a 1 Gyr block from 0-5 Gyr. Diffusion has been implemented in this analysis but not churning, i.e. mixing without heating (Sellwood \& Binney 2002). For the ongoing analysis Chris Flynn has already implemented churning in his simulations.

that escape for stellar cluster that are being disrupted by the influence of the tidal field imposed by the Galaxy. Following these systems in a realistic potential for our Galaxy, i.e. including anisotropic potentials like a bar and spiral arms, will provide insights on the origin of different stellar streams.

\section{Conclusions}

We have focussed on the interpolation along the main sequence, where the relatively smooth behaviour can be reproduced by interpolating in time or H-core abundance. Moving to later stages of the evolution will be more challenging since determining quantities that represent equal evolutionary stages between models is not that straightforward (see Pols et al. 1998).

Once we have a larger grid of stellar models we will be able to replace in a more feasible way the stellar evolution in NBODY6, helping to ensure that this code remains the most advanced direct code in existence.

\section{Acknowledgements}

Guido R. I. Moyano Loyola thanks the LOC for the economical support granted to assit the conference. 


\section{References}

[1] S. Aarseth, Gravitational N-body Simulations, Cambridge Monographs of Mathematics Physics, Cambridge, 2003

[2] K. Freeman, J. Bland-Hawthorn, The New Galaxy: Signatures of Its Formation, 2002, ARA\&A, 40, 487 [astro-ph/0208106]

[3] J. R. Hurley, O. R. Pols, C. A. Tout, Comprehensive analytic formulae for stellar evolution as a function of mass and metallicity, 2000, MNRAS, 363, 293 [astro-ph/0001295]

[4] J. R. Hurley, C. A. Tout, O. R. Pols, Evolution of binary stars and the effect of tides on binary populations, 2002, MNRAS, 329, 897 [astro-ph/0201220]

[5] O. R. Pols, K.P. Schröder, J. R. Hurley, C. A. Tout, P. P. Eggleton, Stellar evolution models for Z = 0.0001 to $0.03,1998$, MNRAS, 298, 525

[6] J. A. Sellwood, J. J. Binney, Radial mixing in galactic discs, 2002, MNRAS, 336, 785 [astro-ph/0203510] 